\title{
The Fatal Outcome of a Child with a Delayed Posterior Urethral Valve Diagnosis in a Low Income Country: A Case Report and Take Home Messages
}

\author{
Francky Teddy A Endomba ${ }^{1^{*}}$, Jan René Nkeck ${ }^{1}$, Joel Noutakdie Tochie ${ }^{1}$, Junette MA Metogo ${ }^{2}$ and Jacqueline Ze Minkande ${ }^{2,3}$ \\ ${ }^{1}$ Department of Medicine and Biomedical Sciences, University of Yaoundé I, Yaoundé, Cameroon \\ ${ }^{2}$ Department of Surgery and Specialties, University of Yaoundé I, Yaoundé, Cameroon \\ 3 Intensive Care Unit, Yaoundé Gynaeco Obstetric and Pediatric Hospital, Yaoundé, Cameroon
}

*Corresponding author: Francky Teddy A Endomba, Department of Medicine and Biomedical Sciences, University of Yaoundé I, P.O. box 1364, Yaoundé, Cameroon, Tel: +237696136647; E-mail: tedissimo@yahoo.com

Received Date: July 17, 2017; Accepted Date: July 20, 2017; Published Date: July 27, 2017

Copyright: (c) 2017 Endomba FTA, et al. This is an open-access article distributed under the terms of the Creative Commons Attribution License, which permits unrestricted use, distribution, and reproduction in any medium, provided the original author and source are credited.

\begin{abstract}
Introduction: When faced with early urinary tract infections in infants, there is a need to do diagnostic imaging to exclude urinary tract malformations. Delayed diagnosis of this condition could lead to serious complications.

Case presentation: We received a 2 months old male infant referred for better management of an acute kidney injury (AKI) with septicemia. He had a past medical history of febrile urinary tract infection. Our diagnosis was that of acute kidney injury and septicemia associated to a posterior urethral valve. The initial treatment included broad spectrum antibiotics, management of $\mathrm{AKI}$ complications and vesicostomy to relief the urinary obstruction prior to valve ablation. We unfortunately lost the infant on day 3 following surgery from severe refractory hyperkalemia and a uremic syndrome.
\end{abstract}

Conclusion: This case is a call to clinicians' attention in low income countries to always check for urinary tract malformations when confronted with an infant presenting a febrile urinary tract infection.

Keywords: Acute kidney injury; Diagnosis delay; Posterior urethral valve

\section{List of Abbreviations}

AKI: Acute Kidney Injury; CALUT: Congenital Anomalies of Lower Urinary Tract; ICU: Intensive Care Unit; PUV: Posterior Urethral Valves; UTI: Urinary Tract Infection

\section{Introduction}

Acute kidney injury (AKI) previously known as acute renal failure is a general term representing a wide range of renal dysfunctions. The Kidney Disease: Improving Global Outcomes (KDIGO) guidelines defines it as "an abrupt reduction in kidney function that includes, but is not limited to, acute renal failure" [1]. It can lead to several acute complications and its long term outcome in case of survival can either be full recovery of the renal function or slow progression towards chronic kidney disease [2,3]. AKI is not only of concern to adults, as the pediatric population can also be affected. Just as in adults, etiologies of pediatric AKI can be classified as pre-, intrinsic- and postrenal $[4,5]$. Although post-renal causes are not the most common, they are associated to the same complications and have as particularity that surgical intervention is required for remission [6]. Posterior urethral valve (PUV) is the most common pediatric obstructive uropathy and equally represents an undermined cause of preventable AKI in children [7]. We hereby present a case of complicated acute kidney injury associated with septicemia, as a complication of posterior urethral valve in a Cameroonian child.

\section{Case Presentation}

We received a 2 months old male infant referred for acute kidney injury (AKI) with septicaemia. His past history was remarkable with a treated urinary tract infection two weeks prior. He was born at term through normal vaginal delivery following an uneventful pregnancy. The present condition began three days prior to his referral by respiratory distress and pallor for which he was hospitalised in a health center for suspected late onset neonatal sepsis and as a result received an empirical antibiotherapy made of Ceftriaxone, Gentamicin and Ampicillin. Due to the lack of improvement in his clinical condition after 48 hours, the antibiotherapy was changed to Imipenem and Amikacine and the child was referred to our hospital for better management. On admission in our hospital, he was conscious and had a temperature of $38.9^{\circ} \mathrm{C}$, heart rate 146 beat per minute, respiratory rate 44 cycles per minute, peripheral oxygen saturation $89 \%$ and urinary output $0.21 \mathrm{ml} / \mathrm{kg} / \mathrm{h}$. Generalised edema, bilaterally enlarged kidneys and a distended urinary bladder were evident on physical examination. The work-up on entry revealed a white blood cell count of 16,100 cells $/ \mathrm{mm}^{3}$, hemoglobin $6.8 \mathrm{~g} / \mathrm{dl}$, potassium $5.85 \mathrm{mmol} / \mathrm{L}$, urea $1.39 \mathrm{~g} / \mathrm{L}$, creatinine $28.23 \mathrm{mg} / \mathrm{L}$, and sodium $123.4 \mathrm{mmol} / \mathrm{L}$. The abdominal ultrasound revealed uretero-hydronephrosis associated with dilatation of the prostatic urethra suggestive of underlining posterior urethral valves (Figure 1). We suspected a posterior urethral valve complicated by septicemia and postrenal acute kidney injury. $\mathrm{He}$ was admitted to the pediatric Intensive Care Unit (ICU) where he was put on a treatment to correct AKI complications, an antibiotherapy and underwent an emergency vesicostomy under general anaesthesia after a blood transfusion. The surgery was uneventful with no intra 
Citation: Endomba FTA, Nkeck RJ, Tochie JN, Metogo JMA, Minkande JZ (2017) The Fatal Outcome of a Child with a Delayed Posterior Urethral Valve Diagnosis in a Low Income Country: A Case Report and Take Home Messages. J Nephrol Ther 7: 297. doi: 10.4172/2161-0959.1000297

Page 2 of 3

operative complication. The initial blood and urine cultures were positive for Klebsiella pneumonia which was sensitive to Imipenem, Ofloxacine, Amikacine, Amoxicilline and Levofloxacin, and led us to maintain the ongoing antibiotherapy. Moreover, his cerebrospinal fluid culture was sterile. The early postoperative period was marked by progressive loss of consciousness, refractory hypernatremia (167.5 $\mathrm{mmol} / \mathrm{L})$ and severe refractory hyperkaliemia $(6.90 \mathrm{mmol} / \mathrm{L})$. The infant died on the day three following surgery.
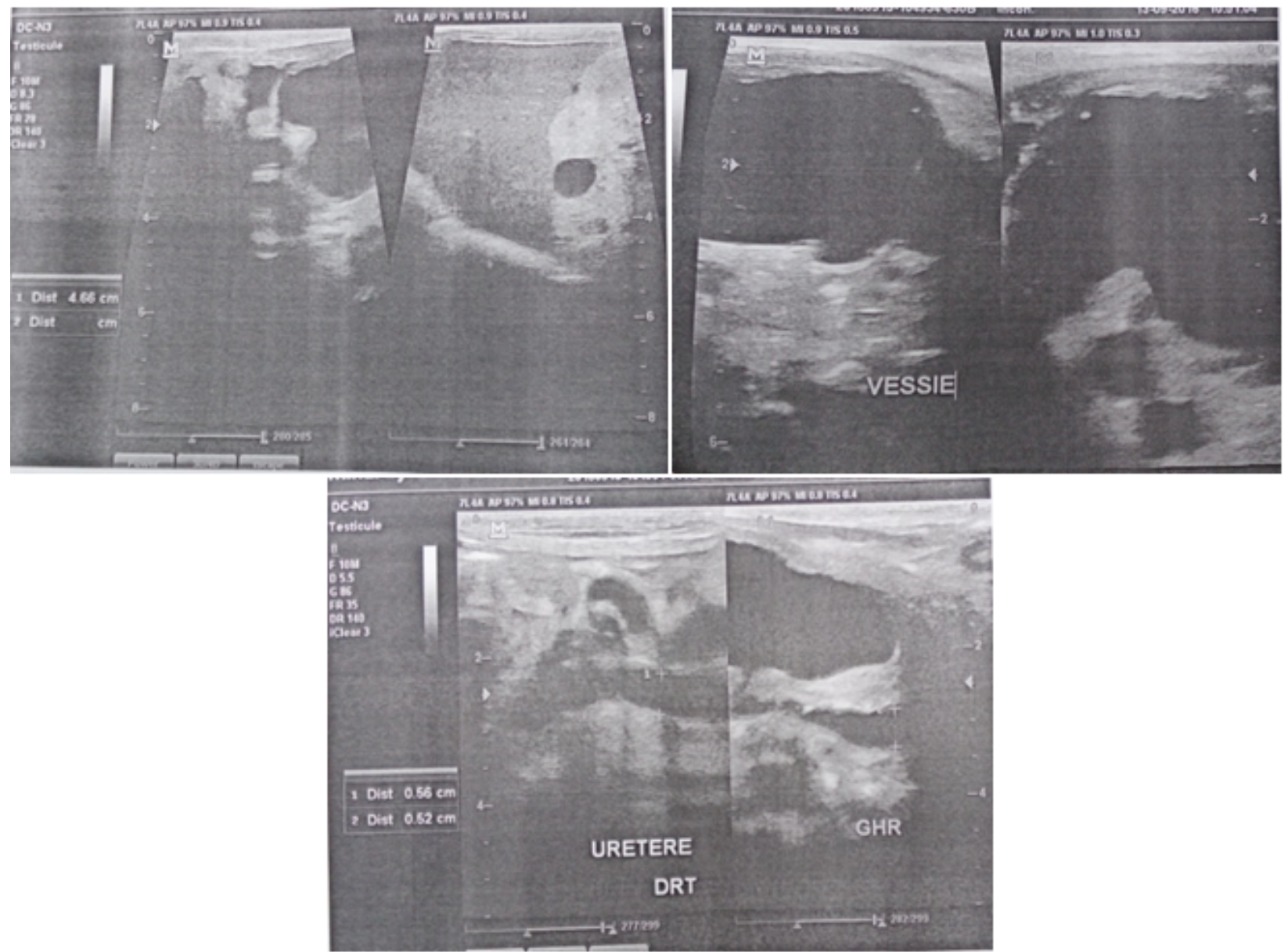

Figure 1: Findings on ultrasonography of the genitourinary system showing bilateral uretero-hydronephrosis, distended bladder and dilation of the prostatic urethra.

\section{Discussion}

Congenital Anomalies of the Lower Urinary Tract (CALUT) is a family of diseases characterised by the presence of a birth defect at the level of ureters and/or bladder and/or the urethra [8]. With respect to urethral anomalies, we can distinguish three types: hypospadias, posterior urethral valves (PUV) and anterior urethra abnormalities $[8,9]$. PUV also referred to congenital obstructive posterior urethral membrane, is the most common cause of lower urinary tract obstruction in children with a predilection for the male gender [10]. Indeed, boys are more prone to this because of some additional developmental processes involved in the formation of the male phallic urethra. PUV, first described by Morgagni in 1717, is defined by the formation of sail-like membrane folds from the verumontanum in the posterior urethra and occurs during an early embryonic stage $[7,8]$. This birth defect occurs in one of every 5000 to 8000 male infant and further leads to urine outflow obstruction and elevated pressures within the urinary bladder, ureters and kidneys $[8,11]$. The pathogenesis of PUV is however still unclearly defined [8]. While this pathology is frequently diagnosed during the antenatal period in highincome countries by an obstetrical ultrasound, diagnosis in lowincome settings is often made in the late postnatal period when complications arise, as observed in our case. In neonates and infants the signs are mostly delayed voiding or poor urinary stream, abdominal mass, poor feeding and failure to thrive $[7,12]$. In the older child, a weak urinary stream and recurrent urinary tract infections (UTIs) are more common [7,13,14]. The American Academy of Pediatrics recommends that morphological exams should be performed in any infant aged between 2 months to 2 years who presents with a febrile urinary tract infection [15]. Ultrasonography is usually the initial imaging modality used when PUV suspected. Sonographic findings often include thick bladder wall, bilateral asymmetric hydronephrosis, and the "keyhole" sign made of the dilatation of the posterior urethra [10]. Although the ultrasound scan of the urinary tract is the most widely used diagnostic tool for PUV, it can be complemented by micturating cystourethrogram and/or urethral endoscopy which is the gold standard for the diagnosis $[9,10]$. 
Citation: Endomba FTA, Nkeck RJ, Tochie JN, Metogo JMA, Minkande JZ (2017) The Fatal Outcome of a Child with a Delayed Posterior Urethral Valve Diagnosis in a Low Income Country: A Case Report and Take Home Messages. J Nephrol Ther 7: 297. doi: 10.4172/2161-0959.1000297

Page 3 of 3

PUV has to be corrected early enough by urethral catheter and cystoscopic fulguration of the valve $[9,10,12]$. However, for several reasons and in some cases like ours, a urinary derivation by vesicostomy or pyeloureterotomy might be realised prior to further valve ablation $[7,9,12]$. Without a precautious therapeutic intervention, as seen in our case, this birth defect can lead to serious complications like AKI, septicemia, renal abscess, septic shock, multisystem organ failure and others $[6,8]$. Of these, one of the most dangerous is acute kidney injury which can easily lead to the death $[4,5]$. This fatal outcome is due to complications such as electrolyte disturbances, mostly hyperkaliemia, uremic syndrome and acid-base disorders, as in our case $[4,14]$. To prevent this outcome in low income country like ours where antenatal diagnosis is rarely done, physicians should be aware of obstructive signs, urinary tract infections in children less than 2 years and do imaging of the urinary tract when it's required.

\section{Conclusion}

The fatal outcome of this case revealed diagnostic delay of a CALUT and draws clinicians' attention to the importance of well-timed morphological investigation requests when confronted with an infant presenting a febrile urinary tract infection.

\section{Declarations}

\section{Consent to participate}

Written informed consent was obtained from the patient's legal guardian(s) for publication of this case report and any accompanying images. A copy of the written consent is available for review by the Editor-in-Chief of this journal.

\section{Author's contributions}

FTAE and JRN: Collection of patient information's and care of the child, JNT and AJMM: Review of the Manuscript, JZM: Supervision.

\section{References}

1. Kidney Disease: Improving Global Outcomes (KDIGO). Acute Kidney Injury Work Group (2012) KDIGO Clinical Practice Guideline for Acute Kidney Injury. Kidney inter 2: 1-138.

2. Ostermann M, Joannidis M (2016) Acute kidney injury: Diagnosis and diagnostic workup. Crit Care 20: 299.

3. Shah SR, Tunio SA, Arshad MH, Moazzam Z, Noorani K, et al. (2015) Acute kidney injury recognition and management: A review of the literature and current evidence. Glob J Health Sci 8: 120-124.

4. Gulati S (2012) Acute kidney injury in children. Clin Queries Nephrol 1: 103-108.

5. Andreoli SP (2009) Acute kidney injury in children. Pediatr Nephrol 24: 253-263.

6. Chevalier RL (2016) Prognostic factors and biomarkers of congenital obstructive nephropathy. Pediatr Nephrol 31: 1411-1420.

7. Hodges SJ, Patel B, McLorie G, Atala A (2009) Posterior urethral valves. Scientific World Journal 9: 1119-1126.

8. Rasouly HM, Lu W (2013) Lower urinary tract development and disease. Wiley Interdiscip Rev Syst Biol Med 5: 307-342.

9. Levin TL, Han B, Little BP (2007) Congenital anomalies of the male urethra. Pediatr Radiol 37: 851-862.

10. Ikuerowo SO, Balogun BO, Akintomide TE, Ikuerowo AOA, Akinola RA, et al. (2008) Clinical and radiological characteristics of Nigerian boys with posterior urethral valves. Pediatr Surg Int 24: 825-829.

11. Krishnan A, de Souza A, Konijeti R, Baskin LS (2006) The anatomy and embryology of posterior urethral valves. J Urol 175: 1214-1220.

12. Casella DP, Tomaszewski JJ, Ost MC (2012) Posterior urethral valves: Renal failure and prenatal treatment. Int J Nephrol 2012: 351067.

13. Bhadoo D, Bajpai M, Panda SS (2014) Posterior urethral valve: Prognostic factors and renal outcome. J Indian Assoc Pediatr Surg 19: 133-137.

14. Ashraf M, Shahzad N, Irshad M, Hussain SQ, Ahmed P (2014) Pediatric acute kidney injury: A syndrome under paradigm shift. Indian J Crit Care Med 18: 518-526.

15. Roberts KB (2011) Urinary tract infection: clinical practice guideline for the diagnosis and management of the initial UTI in febrile infants and children 2 to 24 months. Pediatrics 128: 595-610. 